\title{
The Influence of Hispanic Ethnicity on Nonsmall Cell Lung Cancer Histology and Patient Survival:
}

\author{
An Analysis of the Surveillance, Epidemiology, and End Results Database
}

\section{Scarlett Lin Gomez, PhD,}

Cancer Prevention Institute of California, Fremont, California, Department of Health Research and Policy, Stanford Cancer Center, Stanford University School of Medicine, Stanford, California

Clayton W. Schupp, PhD, and

Cancer Prevention Institute of California, Fremont, California

\section{Manali Patel, MD \\ Department of Medicine, Stanford University School of Medicine, Stanford, California}

The article by Saeed et $\mathrm{al}^{1}$, which demonstrated greater rep-resentation of the bronchioalveolar carcinoma subtype and improved survival after non-small cell lung cancer (NSCLC) among US-born and foreign-born Hispanics compared with non-Hispanic whites, has already begun to change the dialogue and inform future research and potential clinical implications regarding the biology and prognosis of NSCLC among Hispanic patients. However, we have major concerns about their findings. Saeed et al examined survival after NSCLC among US-born and non-US-born Hispanics compared with non-Hispanic whites using population-based data from the Surveillance, Epidemiology, and End Results (SEER) 17-registry database. Among their 18,206 Hispanic patients with NSCLC, Saeed et al described 15,629 (85.8\%) individuals as US-born and 2577 (14.2\%) as non-US-born ${ }^{1}$. However, the authors did not indicate how they derived birthplace, nor did they describe any cases as having unknown birthplace.

With regard to birthplace, SEER data are well documented to be incomplete (ie, unknown) for up to one-third of Hispanic cases, and those without a recorded birthplace have been shown to be more likely to be US-born and alive., ${ }^{2,3}$ These findings have significant implications for the accuracy of survival analyses comparing US-born with foreign-born cases from SEER data. Although Saeed et al did not indicate that they excluded Hispanic cases with an unknown birthplace from their analysis, the nativity distribution of Hispanic cases (with $85.8 \%$ of cases being born in the United States) ${ }^{1}$ is inconsistent with national population data that demonstrated that approximately 55\% of US Hispanics are born in the United States. ${ }^{4}$ Furthermore, applying our validated method for imputing nativity in cancer registry data ${ }^{5}$ to the $10 \%$ of Hispanic NSCLC cases without registry or death certificate birthplace information in the California Cancer Registry (which comprises approximately $50 \%$ of SEER cases) during the same years as the study by Saeed et al ${ }^{1}$, we estimated approximately $51 \%$ of Hispanic patients with NSCLC to be US-born.

Conflict of Interest Disclosures: The authors made no disclosures. 
We are encouraged to note research that acknowledges the heterogeneity of cancer incidence and outcomes across detailed racial/ethnic groups. However, with regard to the article by Saeed et al, ${ }^{1}$ the lack of transparency and full disclosure in the methods, critical use of the data, and integration of relevant literature call into question their results and conclusions. SEER data are well respected and widely used for establishing population-level patterns in cancer incidence and outcomes that, in turn, inform public health and clinical action. Thus, analyses using these data must involve careful attention to data limitations to avoid generating potentially faulty results, which can be significant and far-reaching.

\section{Acknowledgments}

Funding Support: Supported by the National Cancer Institute (NCI)'s Surveillance, Epidemiology, and End Results (SEER) Program under contract HHSN261201000140C awarded to the Cancer Prevention Institute of California. The collection of cancer incidence data used in this study was supported by the California Department of Health Services as part of the statewide cancer reporting program mandated by California Health and Safety Code Section 103885; the NCI's SEER Program under contract HHSN261201000140C awarded to the Cancer Prevention Institute of California, contract HHSN261201000035C awarded to the University of Southern California, and contract HHSN261201000034C awarded to the Public Health Institute; and the Centers for Disease Control and Prevention's National Program of Cancer Registries, under agreement \#1U58 DP000807-01 awarded to the Public Health Institute. The ideas and opinions expressed herein are those of the authors, and endorsement by the State of California, the California Department of Health Services, the NCI, or the Centers for Disease Control and Prevention or their contractors and subcontractors is not intended nor should be inferred.

\section{References}

1. Saeed AM, Toonkel R, Glassberg MK, et al. The influence of Hispanic ethnicity on nonsmall cell lung cancer histology and patient survival: an analysis of the Survival, Epidemiology, and End Results database. Cancer. 2012; 118:4495-4501. [PubMed: 22528551]

2. Gomez SL, Glaser SL. Quality of birthplace information obtained from death certificates for Hispanics, Asians, and Pacific Islanders. Ethn Dis. 2004; 14:292-295. [PubMed: 15132217]

3. Gomez SL, Glaser SL. Quality of cancer registry birthplace data for Hispanics living in the United States. Cancer Causes Control. 2005; 16:713-723. [PubMed: 16049810]

4. Pew Hispanic Center. [Accessed January 21, 2010] Statistical Portrait of Hispanics in the United States, 2008. pewhispanic.org/factsheets/factsheet.php?FactsheetID $=58$

5. Keegan TH, Quach T, Shema S, Glaser SL, Gomez SL. The influence of nativity and neighborhoods on breast cancer stage at diagnosis and survival among California Hispanic women. BMC Cancer. 2010; 10:603. [PubMed: 21050464] 